\title{
Avelumab Maintenance Therapy Improves Survival in Metastatic Urothelial Carcinoma
}

\author{
Powles T, Park SH, Voog E, et al. Avelumab maintenance therapy for advanced or metastatic \\ urothelial carcinoma. N Engl J Med. 2020;383:1218-1230.
}

\section{Study Overview}

Objective. To evaluate the efficacy of maintenance avelumab in patients with advanced urothelial carcinoma who had received first-line platinum-based chemotherapy.

Design. International, open-label, randomized, phase 3 trial.

Intervention. Patients were randomized in a 1:1 ratio to receive either maintenance therapy with avelumab 10 $\mathrm{mg} / \mathrm{kg}$ plus best supportive care (BSC) or BSC alone, per local practice. Randomization was stratified according to best response to first-line chemotherapy and metastatic site (visceral vs nonvisceral). Treatment was continued until progression, unacceptable toxicities, or patient withdrawal occurred.

Setting and participants. A total of 700 patients were enrolled at 197 sites (350 in the avelumab group and 350 in the BSC group). All patients had histologically confirmed unresectable or metastatic urothelial carcinoma. Patients received 4 to 6 cycles of chemotherapy with either gemcitabine plus cisplatin or carboplatin and had no evidence of progression after completion. Patients had a treatment-free interval of 4 to 10 weeks prior to starting maintenance therapy. Patients who received neoadjuvant or adjuvant platinum-based therapy within the prior 12 months were excluded.

Main outcome measures. The primary endpoint was overall survival (OS) assessed in both the overall population and PD-L1-positive population. Secondary endpoints included progression-free survival (PFS), objective response, time to response, duration of response, and disease control. PD-L1 expression was determined via the Ventana PD-L1 assay (SP263), and patients were classified as PD-L1 positive if they met 1 of the following: (1) at least $25 \%$ of tumor cells were positive for PD-L1; (2) at least $25 \%$ of immune cells were positive for PD-L1 if more than 1\% of the tumor area contained immune cells; and (3) $100 \%$ of immune cells stained for PD-L1 if no more than $1 \%$ of the tumor area contained immune cells.

Results. The baseline characteristics were well balanced between the groups. A total of $51.1 \%$ of patients had PD-L1-positive tumors (57.6\% in the avelumab group and $56.3 \%$ in the control group). At the time of analysis, $24 \%$ of patients in the avelumab group were still receiving therapy compared with only $7 \%$ in the BSC group. The most common reason for discontinuation of therapy was disease progression; $43.7 \%$ of patients in the control

\section{Outcomes Research in Review SECTION EDITORS}

KatRina F. MATEo, PhD, MPH

CUNY School of Public Health New York, NY

TAISHI HIRAI, MD

University of Missouri

Columbia, MO
DANIEL ISAAC, DO, MS

Michigan State University

East Lansing, Ml

WiLLIAM HUNG, MD, MPH

Icahn School of Medicine at Mount Sinai

New York, NY
FRED Ko, MD, MS

Icahn School of Medicine at Mount Sinai New York, NY

GORDON NGAI, MD, MPH

Icahn School of Medicine at Mount Sinai New York, NY 


\section{Outcomes Research in Review}

group received anti-PD-1 or anti-PD-L1 therapy at progression. The median follow-up was 19 months. OS at 1 year was $71.3 \%$ in the avelumab group and $58.4 \%$ in the control group. The median OS was 21.4 months in the avelumab group compared with 14.3 months in the control group (hazard ratio [HR] for death, 0.69; confidence interval [Cl], 0.56-0.86, $P=0.001$ ). In the PD-L1-positive population, OS was also significantly longer in the avelumab group (NE vs 17.1 months, $\mathrm{HR}, 0.56$; $\mathrm{Cl}, 0.40-0.79$; $P<0.001$ ). In the PD-L1-negative population, median OS was 18.8 months in the avelumab group versus 13.7 months in the control group (HR, 0.85). PFS was longer in the avelumab group than in the control group, with a median PFS of 3.7 months versus 2 months, respectively. The median PFS was 5.7 months in the avelumab group and 2.1 months in the control group in the PD-L1positive population.

Adverse events (AEs) of any grade occurred in 98\% of patients in the avelumab group and $77 \%$ in the control group. Grade 3 or higher AEs occurred in $47.4 \%$ of patients in the avelumab group. AEs led to discontinuation in $11.9 \%$ of patients in the avelumab group. Two patients died in the avelumab group as a result of toxicity (urinary tract infection with sepsis and ischemic stroke). Immune-related adverse events occurred in $29.4 \%$ of patients in the avelumab group. Of those, $7 \%$ were grade 3 in nature, and there were no grade 4 or 5 immunerelated AEs. The most commonly seen immune-related AEs were thyroid disorders.

Conclusion. Avelumab maintenance significantly improved OS compared with BSC in patients with advanced/metastatic urothelial carcinoma whose disease did not progress after first-line platinum-based chemotherapy.

\section{Commentary}

In summary, the JAVELIN Bladder 100 trial showed significantly longer OS with the use of maintenance avelumab following first-line platinum-based chemotherapy. This survival benefit was seen in all subgroups, including those who received cisplatin or carboplatin therapy, as well as those with stable disease, partial response, or complete response to initial chemotherapy. Furthermore, the survival benefit was seen in both the overall population as well as in the PD-L1-positive population. There did not appear to be any new safety concerns noted in this trial. Based on these findings, avelumab maintenance in those who do not progress on first-line platinum-based therapy certainly represents a potentially new standard of care in this patient population. While the results of this study are promising and potentially practice changing, whether this "switch maintenance" approach is superior to treatment at progression (ie, use of checkpoint inhibition in the second-line setting) remains debatable. Nevertheless, for most patients, this appears to be the preferred approach given the notable longer OS and improved PFS, which is meaningful, particularly if the progression event is symptomatic. Furthermore, a portion of patients will not proceed to second-line therapy for a variety of reasons, and thus will not be exposed to checkpoint inhibitors if one takes a treatment break approach.

In the previous KEYNOTE-45 study evaluating pembrolizumab versus chemotherapy in the second-line setting after progression on previous platinum therapy, the median OS was just 10 months in the pembrolizumab arm. ${ }^{1}$ This is markedly different from the 21.4-month median OS noted in the current study. While there are many limitations to this comparison, it does appear that switch maintenance leads to meaningful improvements in patient outcomes. It should be noted, however, that a portion of patients will have a durable response to platinum-based therapy, and thus there may be a portion of patients who would be "overtreated" with such an approach.

A similar approach has been explored in a randomized phase 2 trial looking at maintenance pembrolizumab after first-line chemotherapy (HCRN GU14-182). ${ }^{2}$ This trial similarly showed improvement in PFS; however, OS was not yet mature at the time of data analysis. It should be noted that crossover was permitted in the HCRN study, while this was not allowed in the current Javelin 100 study. Certainly, this crossover effect influenced OS data in that trial. Thus, the current study is the first and only to show an OS benefit with such an approach in this population. Numerous ongoing studies are seeking to evaluate the efficacy of immune check- 
point inhibitors in the first-line setting for advanced urothelial carcinoma, and the results of these studies will help shed additional light regarding the efficacy of this approach.

\section{Applications for Clinical Practice}

First-line maintenance avelumab in patients who do not progress on platinum-based chemotherapy improves both progression-free and overall survival. This approach is certainly practice-changing and represents a new standard of care in this patient population. Careful discussion with each patient about the benefits and risks of a switch maintenance approach is warranted.

Daniel Isaac, DO, MS

doi:10.12788/jcom.0025

\section{References}

1. Bellmunt J, de Wit R, Vaughn DJ; KEYNOTE-045 Investigators. Pembrolizumab as second-line therapy for advanced urothelial carcinoma. N Engl J Med. 2017;376:1015-1026.

2. Galsky MD, Mortazavi A, Milowsky Ml, et al. Randomized double-blind phase ii study of maintenance pembrolizumab versus placebo after first-line chemotherapy in patients with metastatic urothelial cancer. J Clin Oncol. 2020;38:1797-1806. 